\title{
Editorial: Advanced physical methods in brain research
}

\author{
Roberto Bellotti ${ }^{\mathrm{a}}$ and Saverio Pascazio ${ }^{\mathrm{b}}$ \\ Dipartimento di Fisica and MECENAS, Università di Bari, I-70126 Bari, Italy \\ INFN, Sezione di Bari, I-70126 Bari, Italy
}

Received: 20 November 2012

Published online: 30 November 2012 - (c) Società Italiana di Fisica / Springer-Verlag 2012

\begin{abstract}
The brain is the most complex organ of the human body. It is located in the head and is the center of the nervous system. Its largest part is the cerebral cortex, which contains tens of billions neurons. On average, each neuron is connected to other neurons through several thousand synapses, which pass electrical (or chemical) signals, to form a very intricate and extremely complex system. The brain is estimated to contain from 1 to $5 \times 10^{14}$ synapses.

The cerebral cortex is the main characteristic of the human brain (when compared to the brains of other mammals). It consists of a thick layer of neural tissue, folded in a way that maximizes the surface that can fit into the volume available (the skull, whose thick bones provide the necessary physical protection). Each side of the brain is divided into four sections, or "lobes", called frontal lobe, parietal lobe, temporal lobe, and occipital lobe. Each of them is associated with specific functions. The so-called "executive functions", such as attention, working memory, planning, organisation, as well as reasoning and abstract thought, are located in the more dorsolateral portions of anterior frontal lobes, while more phylogenetically "ancient" functions like emotions, risk evaluation and impulse control are situated in more medial areas belonging to the limbic system. Continuing with a rough subdivision of work: more posterior parts of the frontal lobes are in charge of voluntary movement; parietal lobes of the relationship between body and space (visuo-spatial functions); temporal lobes of memory and language (the latter lateralised to the dominant emisphere), and occipital lobes of vision. Complex association networks integrate information from the various brain regions to produce percept, thought and action.
\end{abstract}

Brain structures and their functions have always fascinated scientists, philosophers and thinkers. What makes the brain special, from a wider perspective, is that its physical properties give rise to mental states and in this sense they generate what philosophers call the mind. Through more than two thousand years of Western philosophy the mind was thought to be separate from the brain. This mind-body dualism can be traced back to Plato [1] and was formulated in its modern connotation by René Descartes five centuries ago [2]. Despite rapid scientific progress, the mind's connection with brain activity is not thoroughly understood and much about how the brain works remains a mystery, even nowadays. The way individual brain cells and synapses cooperate, giving rise to thought and other superior functions, has challenged biology and medicine, as well as physics and computer science.

The approach to human brain damage and disease has been more successful, maybe because they are easier to define than abstract thought. The human brain is susceptible to different types of damage and disease, with different degrees of "visibility" of the pathogenetic pathway. On the one hand, there are obvious dramatic events, such as a blow to the head, a stroke, or poisoning by neurotoxins. On the other hand, there are more insidiously progressive degenerative disorders, such as Parkinson's disease, or Alzheimer's disease: conditions characterised by neuronal death and loss of brain volume. Finally, there are psychiatric conditions, such as schizophrenia and depression, that are also associated with brain dysfunction, although the nature of the brain abnormalities in these conditions is more subtle, and less well understood.

The techniques used to study the brain can be either invasive or non-invasive. For obvious ethical reasons, the former ones are seldom performed with humans. Non-invasive techniques include neuroimaging or electroencephalography. This Focus Point of The European Physical Journal Plus contains four articles and is devoted to selected open problems in neuroscience, with the emphasis on the advanced brain signal and image processing. During the last years, the development of neuroimaging and signal processing has made the visualization and measurement of pathological brain changes in vivo possible, producing a radical change, not only in the field of scientific research, but also in the

\footnotetext{
a e-mail: roberto.bellotti@ba.infn.it

b e-mail: saverio.pascazio@ba.infn.it
} 
everyday clinical practice. Conventional structural magnetic resonance imaging (MRI) provides increasingly detailed "photographs" of the brain, while advanced techniques, such as the diffusion tensor imaging or tractography, also allow the in vivo recontruction of specific pathways as well as quantitative assessments of tissue integrity. Functional MRI, perhaps more seductively, offers a peep on the active processes of the living brain. Two articles of this Focus Point deal with MRI and two with electroencephalography (EEG) signals.

The article entitled Neurodegenerative dementias: from MR Physics lab to assessment room, by S.D. Bruno et al. [3], is a review structured in two parts: a background section, outlining the basic principles of MRI acquisition and image analysis, followed by a discussion of structural MRI applications in neurodegenerative dementias. The latter is divided into three main avenues: detection of changes in cerebral shape and volume, detection of changes in brain connectivity, and methods of nosological classification. This gives an exhaustive overview of structural MRI applications in the two most common neurodegenerative dementias, Alzheimer's disease and fronto-temporal lobar degeneration, to highlight how different disease models raise different imaging challenges.

The article entitled Alzheimer's disease markers from structural MRI and FDG-PET brain images, by A. Chincarini et al. [4], gives an overview of the underlying assumptions and analysis methods that are used to extract clinically meaningful information from neuroimages, focusing on structural MRI and FDG-PET. In particular, it deals with the early diagnosis of Alzheimer's disease and the recognition of subjects at higher risk to develop dementia: this is a very important aspect for the development of increasingly sensitive biomarkers for therapeutic management and pharmaceutical trials.

Although in the last decades the use of magnetic resonance imaging has grown in popularity as a tool for the structural analysis of the brain, electroencephalography is still nowadays an interesting technique for the understanding of brain organization and function. The main advantage is that EEG allows for a direct measurement of the neuronal activity with a time resolution that is fine enough to account for variations of the neuronal activity of the order of a few milliseconds. On the other hand, EEG spatial resolution is very poor, since it is based on a small number of scalp recordings, thus turning the source localization problem into an ill-posed one, in which infinite possibilities exist for the localization of the neuronal generators. This is an old problem in computational neuroimaging, and many methods have been proposed to overcome this localization.

In the article by M. Papadopoulou et al. [5], entitled Mapping the epileptic brain with EEG dynamical connectivity: established methods and novel approaches, the issue of dynamical connectivity in the brain is discussed. The problem is to retrieve the structure of a complex network, in this case of the brain, from the dynamics at his nodes, in this case the electroencephalographic sensors. Several methods are reviewed and applied on a specific case study: the localization of the epileptogenic zone and early seizure detection from the scalp and intracranial electroencephalogram of an epileptic patient. The take-home message is that an approach tracking the evolution of connectivity both in space and time in the framework of information transfer can be a convenient choice to address this important clinical issue [6,7].

Finally, the article entitled Variational Bayesian localization of EEG sources with generalized Gaussian priors, by J.M. Cortes et al. [8], performs a variational Bayesian inference procedure with a generalized Gaussian prior. The proposed model simultaneously yields the estimation of both sources and algorithm parameters. The inclusion of the generalized Gaussian prior is a novelty and it enables one to control the smoothness degree of the estimated sources.

These selected contributions clarify what the technical difficulties inherent to this expanding field of research are, as well the most recent progress. They also highlight the interdisciplinary character of these investigations. The objective of contemporary research is to characterize the structure of the brain, understanding and unveiling its complexity. Such a formidable task will probably require the combined efforts of the scientific method, ranging from physics and chemistry to biology and medicine, but also the feedback from psychology and psychiatry.

The evolution of diagnostic techniques has enabled researchers to comprehend brain activity and study brain damage and disease towards unexplored avenues. It will be of great interest to understand if the rapid evolution of the way human beings interact with their environment [9], in particular virtual reality, will yield measurable modifications of brain functions and of our awareness of them.

\section{References}

1. B. Russell, A History of Western Philosophy (Simon \& Schuster/Touchstone, 1967).

2. R. Descartes, Discourse on Method and Meditations on First Philosophy (Hacket Publishing Company, 1998).

3. S.D. Bruno et al., Eur. Phys. J. Plus 127, 139 (2012).

4. A. Chincarini et al., Eur. Phys. J. Plus 127, 135 (2012).

5. M. Papadopoulou et al., Eur. Phys. J. Plus 127, 144 (2012).

6. V. Brodbeck et al., Brain 134, 2887 (2011).

7. F. Grouiller et al., Brain 134, 2867 (2011).

8. J.M. Cortes et al., Eur. Phys. J. Plus 127, 140 (2012).

9. J. Monod, Le hasard et la nécessité (Editions Du Seuil, 1964). 